\title{
Possible schemes of calculation modeling in a quantum computer
}

\section{Vladimir Voronov}

Irkutsk State Technical University, Irkutsk, Russia; voronov@,istu.edu.ru

Received 21 April 2010; revised 21 May 2010; accepted 26 May 2010.

\begin{abstract}
In the present work a possibility of computation modeling, which should be realized in a real quantum computer, is discussed. In this connection two models of a device, which work is determined by the structure and dynamics of real molecular systems are reported.
\end{abstract}

Keywords: Quantum Computers; Strong-Correlated Systems; Entanglement of States

\section{INTRODUCTION}

The present work deals with a possibility of computation modeling, which should be realized in a real quantum computer. In this connection, the models of a device, which work is determined by the structure and dynamics of real molecular systems are reported.

Recently $[1,2]$ we have put forward an idea, which realization would allow one to overcome, at least, some problems related to the creation of quantum computers, i.e., the idea of molecular Internet. According to this idea, every molecular system with its own set of degrees of freedom and (or) states represents a server having memory of certain volume. This server is used for storage and transmission of the information. The latter is further transformed in computational operations during the solution of specific mathematical task. If to assume that quantum computer has to perform the same operations as the classical one, the necessary memory volume of such a server could be achieved by two (at least, in principal) routes: 1) introduction of maximum amount of atoms playing the role of q-bits into the molecular system; 2) combination of separate molecules in a spatially ordered structure where physical and chemical interactions of any nature can be realized. These interactions will ensure the formation of superpositional coherent states needed for the performance of computational operations. The above approaches are quite reasonable from the viewpoint of the modern theoretical notions on quantum cal- culation problem. But, in our opinion, the ways of this problem solution are beyond the frameworks of the conventional theory. Below we will discuss some general concepts, which can pave the road to new approaches to the problem of quantum computer creation.

\section{MODELING PRESENTMENT OF QUANTUM COMPUTING}

According to the Shor theory [3], a certain number of states remain free in the course pf information input. In principle, these states can be used for the performance of parallel calculations. Since a number of such states can be very large (relative to the corresponding number of q-bits), the tasks which previously seems insoluble become realistic. As applied to the molecular Internet idea, one can correlate the states of quantum particles (q-bits) with real molecular objects with all far-reaching consequences. Thus, keeping the idea of quantum calculations intact, it is possible to impart it a real content. Really, if the quantum states aforementioned are still virtual, the molecular states are manifested themselves in macroworld by many phenomena which reflect quite adequately these states. Therefore, these states become experimentally accessible. The problem is in the correlation of these states with the corresponding mathematical patterns. Hence, the application of these states for the solution of specific task should involve the usage of the information, which is already contained in the molecular structure.

Logic route to the realization of the molecular Internet idea is in combined application of the spatial molecular structure and spin values of q-bits constituting this molecular structure. Really, we have mentioned above that the problem is in the search for an approach which allows quantum systems (due to the peculiarities of structure and/or behavior) to be manifested in the macroworld. Therefore, if one can manage to correlate q-bit spins with molecular structure this will mean the realization of such an approach. Thus, the circle is closed, i.e. there is 
a principal way to the organization of quantum states detection without any distortion of this state. Experimental realization of the approach becomes predictable: combined usage of the information on the molecular system state and spins (q-bits) contained in this system. Further problems of quantum calculations are the matter of technique to some degree, though not very simple.

The experimental investigations carried out by the present time are far from the realization of quantum calculation idea. Indeed, even though the elementary quantum algorithms have been already elaborated using the NMR method [4-6], the possibility of the creation of real quantum computers on the basis of this phenomenon is yet to be proved. The probable application of other physical methods for this purpose is under discussion. In this case, the approach based on the preliminary modeling the operation scheme, which should be realized in a quantum computer (followed with its embodiment in specific electronic schemes), seems to be intriguing. By the moment there are many essential prerequisites for such experiments. Really, the preparation of nano-sized heterostructures enables to obtain a wide range of properties for different kinds of device applications. In its turn, the development of physics and technologies of synthetic nanostructures makes it possible to prepare them in quite wide variety (see [7] and the references therein). That is way the above model route to the creation of quantum calculation processors may be considered as promising.

All the aforesaid allow one to propose the following variant to model the real quantum calculation. Preliminary it is worthwhile to note that according to the current theoretical notions, to reach the goal one should have such devises (as the starting universal logic block), which can perform repeatedly logic operations "NOR" and "controlled NOR". In other words, these devises (logic gates) must possess a property of reversibility. Among such devices is the Toffoli three-bit gate (see, for example, [8,9] and the references therein). If to stay further within the framework of the model proposed, one should create the elementary blocks: gates; each of them must incorporate three q-bits interacting with each other. At the same time, the environment of each q-bit must be suitable for the triggering the calculation procedure. These blocks can be formed by three nano-sized objects containing an electron with a specific spin state. Next, one should model the reversibility property of the three q-bit gate. This can be attained due to the multiple reproduction of the above block from three nanoobjects as well as the application of special commutating devices operating, for example, on the basis of femto-second laser.

This brings up the questions: "To what degree the pro- posed method for the organization of quantum calculations is realistic and what are the dimensions of the devise capable of such operations as quantum calculations?" If one is limited here by the qualitative answers to the questions arisen it would be reasonable to pay the attention to the germanium and silicon nanostructures prepared recently using scanning tunnel microscope [7]. In this case, one can grow the nanoobjects (islands of approximately $10 \mathrm{~nm}$ size at the base and some $\mathrm{nm}$ in height) located in about $50 \mathrm{~nm}$ from each other. It is an easy to evaluate that every square centimeter will contain about $10^{10}$ of such islands. Therefore, from the nanoobjects mentioned one can create (at least, in principle) the appropriate number of the gates (by collecting the islands in groups) to make up the processor, capable of solving the real tasks.

\section{MODELING OF QUANTUM CORRELATION}

Another step of the quantum calculations needs to be modeled. We mean here the entanglement of states or quantum correlation of the q-bits system localized in nano-islands. This is a mandatory step preceding the calculations [8,9]. Therefore, one can speak about preparation of the entangled state. To model the above step, it is proposed to use the self-organization of quantum (molecular) systems. In other words, one should synthesize the molecules where the entanglement of states is provided due to the self-organization of atoms. Such a possibility could be evaluated as follows. Let the dependence of deviation amplitude $f(x)$ on the initial state $x$ is defined by the expression:

$$
f(x)=a x-b x^{n},
$$

where $a$ and $b$-are constant positive coefficients, $n \geq 2$. If $x<<1$, then $b x^{n}<<a x$, therefore

$$
f(x) \approx a x
$$

Thus, in the case of (2), $f(x)$ grows linearly with growth of $x$. If $x$ value is comparable with 1 , it would be impossible to neglect the $b x^{n}$ member, since for the description of the system behavior one should use the initial Equation (1). Hence, the growth of function deviation at the expense of $a x$ member will cause nonlinear limitation owing to the deduction of $b x^{n}$ value. Under several $x$ values, the $f(x)$ function will be close again to zero and all starts from the beginning. The system will automatically regulate itself, as its properties depend on a current state (in this case-from $x$ value). Therefore, it is reasonable to conclude that by somehow changing the given molecular system, it is 
possible to achieve (in principle) the necessary values for the corresponding parameter (or parameters). Obviously, such changes could be carried out by three ways: 1) the introduction of new atoms to molecular structure; 2) the external action (for example, applying an electromagnetic field); 3 ) the combination of these two ways.

The fundamental problem is to understand what molecular objects can be suitable for similar manipulations. Based on the most general ideas, one can assume that here one should deal with the compounds where characteristic intramolecular interactions will ensure the realization of the required (from a position of a problem discussed here) quantum states. These are the compounds containing atoms with unfilled $3 d-, 4 f-$ and $5 f-$ shells. In solids, such atoms retain localized magnetic moments completely or partially. The strong interaction of electrons of these groups with each other or with collective electrons of outer shells represent a peculiarity imparting the unique properties to a variety of the compounds containing atoms of transition and rare-earth elements. The investigations carried out over the last fifteen - twenty years have shown that for these compounds the diverse physical phenomena are possible. Among them are phase transitions resulting in the magnet-ordered phases and superconductivity, dielectric and metal states; transitions with appearance and disappearance of the localized magnetic moments. The specified properties are the consequence of strong interactions of electronic and magnetic degrees of freedom. The systems with strong interaction of electrons are referred to as the strong-correlated systems [10].

Particularly, the functional dependence (1) can follow to any parameter, which is characteristic for the given molecular system. It is only necessary that the parameter would periodically undergo such changes that its limiting values would correspond to those quantum states which should be used for modeling the process of the quantum calculations. These states can be realized for the molecular systems which behavior at the macroworld level (in laboratory system of coordinates) is connected with the manifestations of heterogeneity of intra-molecular processes. Just due to the peculiarities of structure and dynamics of the molecular system, the nonlinearities, which will provide periodicity in change of a parameter characterizing its behavior, becomes possible. Apparently, a vivid illustration of the possibility of the specified process realization is the compounds belonging to a new class of polynuclear heterospin complexes containing the ligands with non-paired electron [11]. For example, this is tetranuclear nickel cluster $\left[\mathrm{Ni}_{2}(\mathrm{OH})_{3}\right.$ $\left.\left(\mathrm{C}_{5} \mathrm{H}_{9} \mathrm{O}_{2}\right)_{5}\left(\mathrm{C}_{5} \mathrm{H}_{10} \mathrm{O}_{2}\right)_{4}(\mathrm{~L})\right] 1.5 \mathrm{C}_{7} \mathrm{H}_{8}(\mathrm{I})$, where $L-$ is a nitroxide radical. The compound (I) is a representative of the aforementioned strongly correlated systems.

According to the data reported in the work [11],
spin-Hamiltonian, describing the exchange magnetic interactions in these compounds, looks as follows:

$$
H=-2 J J_{0} S_{1} S_{2}-2 J_{1}\left(S_{1} S_{3}+S_{2} S_{4}\right)-2 J_{2}\left(S_{1} S_{2}+S_{2} S_{3}\right),
$$

where $S$ with the corresponding index is a spin value of unpaired electrons localized on $\mathrm{Ni}(I I)$ atoms and nitroxyl groups, $J-$ is a parameter characterizing interaction between the mentioned groups. Naturally, in the case of other compounds which differ from compound (I) in structure, the right part of the expression (3) will look otherwise. However, it is important that by selecting the appropriate initial compounds one can obtain such heteronuclear complex where the interaction of groups having unpaired electron spins will provide the fulfillment of the condition (1).

The question is arisen: "What is nature of quantum object state allowing the model of entanglement to be realized?" To answer the question, one should take into account the following considerations. The description of quantum states, based on the application of spin-Hamiltonian (3) assumes the availability of free (real) quantum particles, spin carriers $S$. Meanwhile, the introduction of unpaired electron into the ligand molecule can lead to the appearance of states caused by the interaction of spin $S$ with electrons of the ligand molecule. It is known that the description of such a collective behavior is often associated with the use of the quasi-particle concept. For example, the notions on magnetic polaron or ferron turned out to be fruitful for the explanation of different heterogeneous charge and spin states in magnets [12]. We will start from the established fact, i.e. from the localization of unpaired electron spins in two positions like in paramagnetic heterospin complexes of elements of the first transition group bearing stable iminoxyl radicals [11]. In the case of unpaired electron spins related to the central ion, with which the ligand molecules are coordinated, it is a common knowledge that the behavior of the spins mentioned can be unambiguously described accounting for $3 d$-orbitals of a metal. We will address therefore to unpaired electrons which are localized on ligands.

Let us consider a simple case: the presence of unpaired electron localized on a ligand. Obviously, this electron will somehow with an electronic subsystem of the ligand molecules. Indeed, the electronic structure of a specific ligand molecule was formed in the absence of unpaired electron. Therefore, the latter should be considered as a certain excitation. Further on, let us admit that that owing to peculiarities (in this case, the specification is not important) of spatial and electron structure of the complex and under the conditions of thermodynamic equilibrium (set by the sample temperature), 
ferron-like states are realized in ligands. Here it is necessary to explain that full spin of ferron state is formed by the local spin and electron spin (see [12] and the references therein). As to the local spin is concerned, it is caused by magnetic (in particular, antiferromagnetic) state of the matrix, where the conductive electron moves. In other words, there is always specific information on the state of such a matrix.

Formally, in the case of heterospin complexes discussed here, a matrix, in which the unpaired electron with spin $S=1 / 2$ is inserted, is a ligand molecule and not solid phase of microsample as it is observed in manganites [12]. The assumption on realization of ferronlike states should mean that the result of the interaction of unpaired electron with electron subsystem of the ligand will be the state with non-compensated electron spin which differs from the state of free electron. In our case, the quantum objects employed in the calculations are electrons. The emergence of ferron states allows one to distinguish electrons transferred to these states for a while. For a certain time, an electron is located on $d$-, or $f$-orbitals of the central ion (for example, nickel). Further, due to the specifics of intramolecular spin-spin interactions the electron is temporarily transferred to ferron state. In a certain period of time (typical for ferron), the electron is again located on metal orbitals. Then the cycle is repeated. Here, once should stress once again that the ferron state differs from the free electron state. Really, it is a common knowledge that a peculiar feature of the objects called quasi-particles is the difference of their mass from the mass of the corresponding free particle. So, the mass of polyaron is hundrets times higher than that of free electron. This feature is also inherits in ferrons [12]. As was mentioned above, the full spin of ferron state also differs from spin of free electron. Thus, the state of entanglement, needed for operation of a quantum computer, turns out to be modeled. The possible route to a large number of quantum objects has been described above.

One should explain why heterospin complexes can be considered as possible objects to model quantum calculations. These are paramagnetic systems containing as ligands the molecular systems having unpaired electron or unpaired electrons. The role of $d-$ or $f$-metal orbitals here is two-fold. Their population with unpaired electrons participating in intramolecular spin-spin interactions ensures the validity of the expression (1). Besides, it is just unpaired electron on these orbitals, being free relative to its magnetic properties, is transferred for a while to other state needed for modeling the entanglement of quantum particle. By changing the structure and composition of a paramagnetic compound as well as the external conditions one can control the entanglement degree of ferron state and state of a free electron, thus controlling also the calculation procedure.

\section{CONCLUSIONS}

In conclusion, one should make the following remarks. The experimental investigations carried out by the present time are still far from practical realization of the quantum calculations idea. Therefore, while the suitable objects for realization of quantum computers idea will be searched, certain intermediate variants could appear. In such a situation, an approach based on preliminary modeling the operations which should be realized in a real quantum computer with their subsequent application in specific electronic schemes, seems to be attractive. For this purpose the compounds containing the atoms with vacant $3 d-, 4 f-$ and $5 f-$ shells could be suitable. We mean here the compounds (for example, heterospin complexes), which unique properties are caused by strong interactions of electronic and magnetic degrees of freedom, i.e. strongly correlated systems. Among these compounds could be, in particular, heterospin systems, i.e. the complexes of paramagnetic ions of transition metals with organic radicals, since spin-spin coupling between unpaired electron spin of different paramagnetic centers of the molecule is typical for such objects. The coupling mentioned can result in phase immiscibility to afford heterogeneous spin states. In its turn, such states can be used for modeling the processes characteristic of quantum computer.

\section{REFERENCES}

[1] Voronov, V.K. (2005) Possible approach to the solution of quantum computers problem. Quantum Computers \& Computing, 5(1), 3-6.

[2] Voronov, V.K. (2006) NMR and the problem of quantum computer creation: New outlook. In: Susan Shannon, Ed., Trends in Quantum Computing Research, NOVA Publisher, New York, 73-90.

[3] Shor, P.W. (1994) Algorithms for computation: Discreta logarithms and factoring. Proceeding of the 35th Annual Symposium on the Foundation of Computer Science, Los Alamitos, CA, USA, 1994, 124-134.

[4] Jones, J.A. and Mosca, M.J. (1998) Implementation of a quantum algorithm on a nuclear magnetic resonance quantum computer. Journal of Chemical Physics, 109(5), 1648-1653.

[5] Chuang, I.L., Gershenfeld, N. and Kubinec, M. (1998) Experimental implementation of fast quantum searching. Physical Review Letters, 80(15), 3408-3411.

[6] Vandersypen, L.M.K., Steffen, M., Breyta, G., Yannoni, C.S., Sherwood, M.H. and Chuang, I.L. (2002) Experimental realization of Shor's quantum factoring algorithm using nuclear magnetic resonance. Nature, 414, 883-887.

[7] Shklyaev, A.A. and Ichikawa, M. (2006) Germanium and 
silicon nanostructure fabrication using a scanning tunneling microscope tip. Uspekhi Physics Nauk, 176(9), 913-930.

[8] Kilin, S.Ya. (1999) Quantum information. Uspekhi Physics Nauk, 169(5), 507-526.

[9] Valiev, K.A. (2005) Quantum computers and quantum computing. Uspekhi Physics Nauk, 175(1), 3-39.

[10] Izyumov, Yu.A. and Kurmaev, È.Z. (2008) Strongly electron-correlated materials. Uspehi Physics Nauk, 178(1), $25-60$.
[11] Ovcharenko, V., Fursova, E., Romanenko, G., Eremenko, I., Tretyakov, E. and Ikorski, V. (2006) Synthesis, structure and magnetic properties of (6-9)-Nuclear $\mathrm{Ni}(\mathrm{II})$ trimethylacetates and their heterospin complexes with nitroxides. Inorganic Chemistry, 45(14), 5338-5350.

[12] Kagan, M.Yu., Klaptsov, A.V., Brodskii, I.V., Kugel, K.I., Sboichakov, A.O. and Rakhmanov, A.L. (2003) Small scale phase separation and electron transport in manganites. Uspekhi Physics Nauk, 173(8), 877-883. 